\title{
RECTIFICATIONS DE NOMENCLATURE
}

1. D'après une lettre de Froilano de Mello, Herpetomonas hemidactyli Mackie, Das Gupta et Swaminath (ces Annales, VII, 1929, p. 349) tombe en syr:mymie avec $H$. hemidactyli F. de Mello et Crisna Suctancar, de l'intestin d'Hemidactylus brookei Gray, à Nova Goa (Inde portugaise) (Bull. Soc. pathol. exot., XV, 1922, p. 795).

2. D'après une lettre de B.-J. Collins, l'espèce type du genre Oropsylla Wagner et Joff, 1926, n'est pas O. ilovaiskii Wagner et Joff, 1926 (ces Annales, VI, 1928, p. 374), mais O. silantiewi (Wagner, 1898).

\section{ERRATUM}

P. 111, au lieu de Tencacularia michiæ, lire : Tentacularia michiæ.

P. 151, ligne 4, au lieu de En Europe, lire : En Pologne.

P. 222, au lieu de Falceustra catesbeianæ, lire : Falcaustra catesbeianæ.

P. 144, première rangée de figures, première figure à droite, au lieu de $\mathrm{K}$, lire : $\mathrm{A}$; deuxième rangée, deuxième figure à gauche, au lieu de $\mathrm{A}$, lire : K. Dans la légende, après Cercaria: Lepoderma-Plagiorchis, ajouter : ?

Le Gérant: F. Amirault.

Cahors, Imprimerie CouesLant (personnel intéressé). - 39.997 